\title{
Multifunctional diffractive optics for optoelectronic system packaging
}

Yeshaiahu Fainman, Fang Xu, Rong-Chung Tyan, Pang Chen Sun, Joseph Earl Ford, et al.

Yeshaiahu Fainman, Fang Xu, Rong-Chung Tyan, Pang Chen Sun, Joseph Earl Ford, Ashok V. Krishnamoorthy, Axel Scherer, "Multifunctional diffractive optics for optoelectronic system packaging," Proc. SPIE 3348, Optical Information Science and Technology (OIST97): Computer and Holographic Optics and Image Processing, (10 March 1998); doi: 10.1117/12.302480

Event: Optical Information Science and Technology, 1997, Moscow, Russian Federation 


\title{
Multifunctional Diffractive Optics for Optoelectronic System Packaging
}

\author{
Y. Fainman, F. Xu, R. Tyan, P.C. Sun, J.E. Ford ${ }^{\dagger}$, A. Krishnamoorthy ${ }^{\dagger}$, and, A. Scherer ${ }^{*}$ \\ Department of Electrical and Computer Engineering,, University of California, San Diego \\ La Jolla, CA 92093-0407 \\ $\dagger$ Bell Laboratories, Lucent Technologies, Holmdel, NJ 07733 \\ * Department of Electrical Engineering, California Institute of Technology, Pasadena, CA 91125
}

\begin{abstract}
Novel diffractive optical elements (DOE) with multifunctionality in polarization or color are reviewed. We review three technological approaches for construction of such DOEs with multifunctionality in polarization: the two-substrate birefringent computer generated hologram $(\mathrm{BCGH})$, the multiple order delay $\mathrm{BCGH}$, and the form birefringent computer generated hologram (FBCGH) approaches. We also discuss the accurate design of such DOEs enabled by our modeling tools based on rigorous coupled wave analysis. Microfabrication techniques developed for realization of these three types of polarization selective DOEs are described. The developed DOEs with multifunctionality in polarization or color are used to package a 3-D optoelectronic VLSI chip, a transparent optical multistage interconnection network, and a wavelength division demultiplexer, providing mechanical and thermal stability, light efficiency, reduced volume, weight, and cost, and increased reliability.
\end{abstract}

\section{INTRODUCTION}

Diffractive optical elements (DOE's) have proven useful in a wide range of applications including data storage ${ }^{1}$, displays ${ }^{2}$, optical interconnection networks ${ }^{3}$, and optoelectronic system packaging ${ }^{4}$. For many applications such DOEs are designed using computer generated holography (CGH) and fabricated as multi-level phase kinoforms leveraging well established microfabrication technology developed for microelectronics. Many of these applications will benefit from DOEs that can perform different functions depending on the different degrees of freedom of the electromagnetic wave, such as polarization and color, allowing more efficient volume utilization. For example, consider a polarization selective $\mathrm{CGH}$, i.e., a general purpose DOE that utilizes polarization as a degree of freedom to encode more information into the same optical element ${ }^{5}$. Such element provides independent impulse responses for the two orthogonal linear polarizations of light. The conventional method to achieve such dual functionality in a free-space optical system requires polarization beam splitters to separate the incident beam into separate spatial paths for each polarization, two different sets of DOEs and optics to compensate for the free-space propagation, and finally a polarization beam splitter to combine the two spatial path at the output (see Fig. 1). Such implementation will result in a bulky and complex optical system imposing stringent alignment tolerance requirements, mechanical and thermal stability in a large volume, etc.

The main objective of multifunctional diffractive optics is to combine the functionality of bulky optical systems into a single DOE, removing most of the system alignment procedures, improving the light efficiency, significantly reducing the volume and the weight, and miniaturizing the system. For example, in an optical disk readout system, a polarization selective CGH element can separate the spolarized input beam from the p-polarized output beams. Therefore, it can be used to replace a pair of conventional polarization insensitive DOEs and a polarization beam splitter cube, (which are currently used in some compact disc (CD) players $^{6}$,) to make the optical head more compact, lighter, and less complex. Our approach combines these three components into a single element and may yield a higher alignment/tracking speed, a more reliable readout system, and a lower system cost. 
Free-space optical systems employing multifunctional DOEs are expected to outperform systems packaged with conventional techniques in terms of mechanical and thermal stability, light efficiency, volume, weight, reliability, and cost.

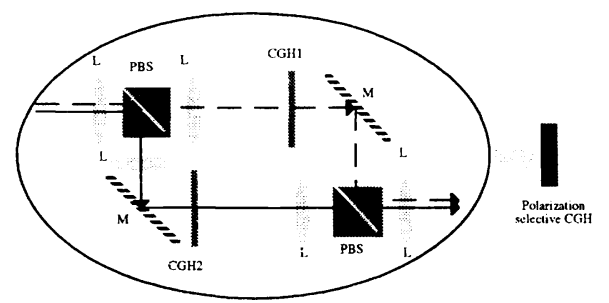

Fig. 1 A polarization selective DOE collapses the functionality of a bulky free-space optical system into a compact optical element .

Conventional DOEs made of isotropic dielectric materials with relatively large features are insensitive to polarization. Specially designed DOEs made of metallic or dielectric gratings with their period slightly less than or comparable to the wavelength of light can be made sensitive to the light polarization, but they will exhibit losses due to absorption or diffraction ${ }^{7}$. Construction of a general purpose high efficiency DOE with multifunctionality in polarization or color will require the availability of additional degrees of freedom for the element design and fabrication. In the next section we will review three technological approaches for construction of DOEs with multifunctionality in polarization: the two-substrate birefringent CGH (BCGH), the multiple order delay (MOD) BCGH, and the form birefringent CGH (FBCGH) approaches. Section 2 will also discuss the accurate design of such DOEs enabled by our modeling tools based on rigorous coupled wave analysis. Section 3 will summarize the microfabrication techniques developed for realization of these three types of polarization selective DOEs. Applications of this technology in image processing, packaging a 3-D optoelectronic VLSI chip, constructing a transparent optical multistage interconnection network (TOMIN), and building a wavelength division demultiplexer will be described in Section 4, followed by the summary and conclusions in Section 5.

\section{DESIGN AND MODELING OF MULTIFUNCTIONAL DOEs}

To make a diffractive optical element with independent and arbitrary impulse responses for two orthogonal linear polarizations, the element must implement two independent phase functions. We developed three different approaches to construct such multifunctional DOEs. Rigorous coupled wave analysis (RCWA) is also applied to the these elements to accurately model performance.

\subsection{Two substrate approach}

If we fabricate a conventional CGH in a birefringent substrate, we could etch the substrate to obtain the correct phase delay for only one of the two possible independent input polarizations. The hologram "seen" by the orthogonal polarization would generally be incorrect. We therefore introduce a second phase only CGH fabricated in a different substrate and place the two surface relief patterns in contact as shown in Fig. $2^{8}$. If the gap between the two substrates and the etch depths are considerably smaller than the feature size, then the two surface profiles can be treated as if they are lying in the same optical plane. These two surface relief patterns give us two degrees of freedom, the independent etch depths $d_{1}$ and $d_{2}$, to satisfy the two requirements of the relative phase difference among every pixel and phase difference between the two orthogonal polarizations inside each pixel. In general, the two substrates can be made of two different birefringent crystals. The gap media can also be birefringent (i.e., liquid crystal) but would normally be isotropic (i.e., air).

Fig. 2 shows a single pixel of a two substrate BCGH. Three special cases of the general BCGH configurations that provide the needed degrees of freedom include: (i) an isotropic gap between a birefringent and an isotropic substrate, (ii) an isotropic gap between two different birefringent 
substrates and (iii) a birefringent gap medium between two different birefringent substrates. In our experiments we choose to make the BCGH using an isotropic gap medium of index $n_{g}$ between two substrates made of same uniaxial material but with crystal axes rotated by $90^{\circ}$ with respect to each other. With this approach we can fabricate both substrates simultaneously with a total number of $2 \log _{2} \mathrm{~N}$ different binary masks and etch steps.

If the refractive index of the gap material, $n_{g}$, matches one of the two indices, e.g., $n_{e}$, then the surface relief "disappeares" for ordinary polarization at the first substrate and for extraordinary polarization at the second substrate. Using this approach, a high performance BCGH element made of calcite was recently demonstrated ${ }^{9}$. The advantages of this approach are: (i) no accurate alignment is required between the two diffractive surfaces and (ii) the etch depth accuracy requirement is greatly reduced because the phase delay caused by etched pixels are less than $2 \pi$.

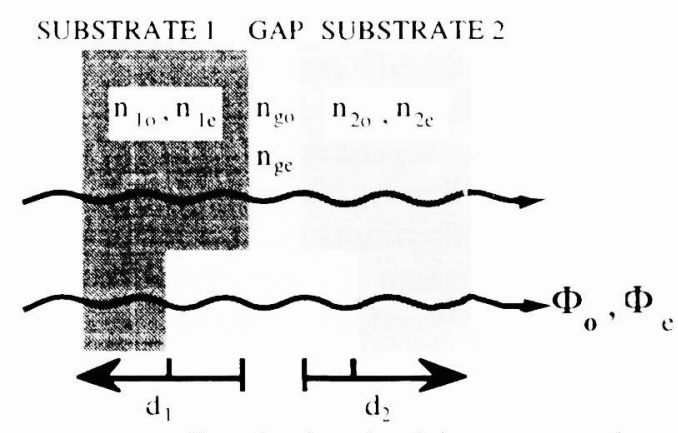

Fig. 2 The geometry of a single pixel in a two substrate BCGH

\subsection{Multiple order delay BCGH}

An alternative approach to achieve polarization selectivity is based on multiple periods of phase delays using a single birefringent substrate (also called modular $2 \mathrm{~m} \pi$ ), as first proposed in [8] and later in [10]. Such an approach may reduce the cost and simplify the fabrication process of polarization selective diffractive optical elements. The required etch depth is deeper than that in a normal DOE. We call such elements multiple order delay (MOD) holograms. Considering a surface relief microstructure fabricated on a birefringent substrate with optic axis parallel to the surface of the substrate, we can find the corresponding phase delays caused by the surface relief compared to an unetched pixel for the ordinary and extraordinary polarized waves, yielding

$$
\begin{aligned}
& k_{0}\left(n_{o}-n_{t}\right) d=2 p \pi+\Phi_{0}^{\prime}, \\
& k_{0}\left(n_{e}-n_{t}\right) d=2 q \pi+\Phi_{e},
\end{aligned}
$$

where $\Phi_{0}+2 \mathrm{p} \pi$ and $\Phi_{\mathrm{e}}^{\prime}+2 \mathrm{q} \pi$ are the phase delays exhibited by ordinary and extraordinary waves; $\mathrm{k}_{0}$ is the wave-vector of the incident wave in vacuum, $n_{o}$ and $n_{e}$ are the refractive indices for ordinary and extraordinary polarized light, respectively, $\mathrm{n}_{\mathrm{t}}$ is the refractive index of the material surrounding the microstructure, $\mathrm{p}$ and $\mathrm{q}$ are integers corresponding to the multiple periods of phase delays exhibited by the ordinary and extraordinary polarized light. In general, Eqs. (1) do not have a unique accurate solution for $\mathrm{d}$ if $\Phi_{\mathrm{o}}$ and $\Phi_{\mathrm{e}}$ are arbitrarily specified design values unless the refractive indexes $n_{0}$ and $n_{e}$ can be controlled in every pixel of the diffractive element as for form birefringent artificial dielectric nanostructures ${ }^{11}$. However, for our design with a homogeneous birefringent substrate with constant values of $\mathrm{n}_{\mathrm{O}}$ and $\mathrm{n}_{\mathrm{e}}$, there exist approximate solutions for $d$ when the values of integers $\mathrm{p}$ and $\mathrm{q}$ are arbitrarily large such that

$$
\begin{aligned}
& \mathrm{k}_{0}\left(\mathrm{n}_{\mathrm{o}}-1\right) \mathrm{d}=2 \mathrm{p} \pi+\Phi_{\mathrm{o}}+\delta_{\mathrm{p}}, \\
& \mathrm{k}_{0}\left(\mathrm{n}_{\mathrm{e}}-1\right) \mathrm{d}=2 \mathrm{q} \pi+\Phi_{\mathrm{e}}+\delta_{\mathrm{q}},
\end{aligned}
$$

are satisfied. In Eq. (2), $\delta_{\mathrm{p}}$ and $\delta_{\mathrm{q}}$ are small numbers introduced to account for the errors and air is assumed to be the material surrounding the microstructure, $\mathrm{n}_{\mathrm{t}}=1$. If the errors introduced by the approximation (i.e., $\lambda \delta_{\mathrm{p}} / 2 \pi\left(\mathrm{n}_{\mathrm{O}^{-1}}\right)$ and $\lambda \delta_{\mathrm{q}} / 2 \pi\left(\mathrm{n}_{\mathrm{e}^{-1}}\right)$ ) are much smaller than the phase steps $\Phi_{\mathrm{p}}$ and $\Phi_{\mathrm{q}}$, 
respectively, we consider this to be a valid approximate solution to Eqs. (1). With this approach, it is possible to apply an arbitrary phase with some specified accuracy to each pixel at each polarization. Therefore, independent multilevel phase holograms can be implemented for the two polarizations. In this approach, the two degrees of freedom come from the etch depth $\mathrm{d}$ and the multiple phase delay orders $\mathrm{p}$ and $\mathrm{q}$.

The ideal substrate material for this application should have large birefringence to assure that the required etch depth $\mathrm{d}$ can be fabricated with high accuracy. We used $\mathrm{x}$-cut yttrium orthovanadate $\left(\mathrm{YVO}_{4}\right)$ grown by CASIX, Inc. as the birefringent substrate. The refractive indices are $\mathrm{n}_{\mathrm{o}}=2.0239$ and $\mathrm{n}_{\mathrm{e}}=2.2600$ at $0.5145 \mu \mathrm{m}$ wavelength. We can find all the possible combinations of $\Phi_{\mathrm{O}}$ and $\Phi_{\mathrm{e}}$ necessary for construction of a binary phase level MOD BCGH ${ }^{12}$. Approximation errors are controlled within $5 \%$ for all cases. Two distinct etches are needed to construct a binary phase level MOD BCGH.

Color selective $\mathrm{CGH}$ : Using the same approach as the MOD BCGH, a wavelength selective $\mathrm{CGH}$ was demonstrated using regular BK7 glass ${ }^{13}$. For color selective CGH we use the substrate's color dispersion degree of freedom as opposed to the polarization dispersion (i.e., birefringence) degree of freedom in the MOD BCGH. The element is constructed to transmit $1.3 \mu \mathrm{m}$ light straight and deflect $1.55 \mu \mathrm{m}$ light at an angle. The first order efficiency of the fabricated element was measured to be $39 \%$ with a $30: 1$ contrast ratio.

\subsection{Form birefringent computer generated holograms (FBCGH)}

BCGHs described above utilize birefringence of the substrates to make the element sensitive to the polarization of light. Another new approach is to create a form birefringent nanostructure and modulate the refractive index and the birefringence at each pixel of the BCGH.

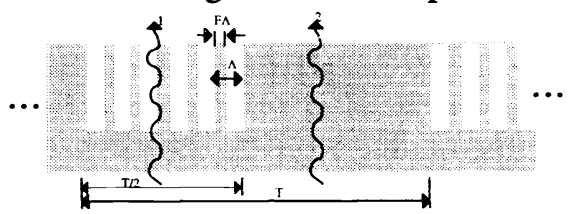

Fig. 3 Design of a form birefringent CGH: one period in a binary phase level FBCGH.

Artificial dielectric materials have been used to construct polarization insensitive ${ }^{14}$ and polarization sensitive DOEs ${ }^{15}$. We designed, fabricated, and experimentally evaluated a binary phase level BCGH element using a form birefringent structure fabricated on bulk GaAs substrate (or form birefringent CGH, FBCGH). The element is designed to transmit TE polarized light straight and deflect TM polarized light at an angle. This is a simplified case of the general FBCGH element.

Fig. 3 shows a single period of a FBCGH element. In this period, one pixel consists of a high spatial frequency grating (HSFG) and the other pixel is just the substrate material. The HSFG does not introduce propagating diffraction orders other than the zero order due to its subwavelength nature. The diffractive structure, on the other hand, introduces many diffraction orders. When the wavelength is much larger than the period of the HSFG, second order effective medium theory (EMT) can be used to calculate the effective indices with high accuracy. The effective indices for TE and TM polarizations are functions of fill-factor and period of the HSFG. The two degrees of freedom needed to encode two phase functions come from the etch depth $d$ and the controllable effective indices for the two orthogonal polarizations. The effective indices can be found using second order EMT. The design obtained using EMT and geometric optics approximation was also verified using RCWA. The two designs were found to be in good agreement.

\subsection{Accurate modeling using rigorous coupled wave analysis}

Designs of multifunctional DOEs described above are based on scalar theory. However, studies show that for a conventional polarization insensitive DOE, when the feature size is 14 times less than the wavelength, scalar theory designs and actually fabricated devices differ in diffraction efficiency 
performance by more than $5 \% 16$. In these cases, rigorous theory has to be applied in order to get more accurate prediction. Moreover, we found that for a two substrate BCGH element, this criteria on the validity of scalar theory extends to features which are 32 times less than the wavelength. When modeling FBCGH elements, rigorous theory becomes essential especially when the period of the diffractive element is comparable to the wavelength of the incident light.

We have applied rigorous coupled wave analysis ${ }^{17}$ to the two substrate BCGH elements as well as to FBCGH elements. The modeling results obtained from rigorous theory show that small fabrication error in the two substrate BCGH elements may cause a large efficiency degradation. The rigorous simulation results also indicated that inter-pixel crosstalk inside a two substrate BCGH caused by deep etch depth and high substrate reflectivity degrades the performance significantly.

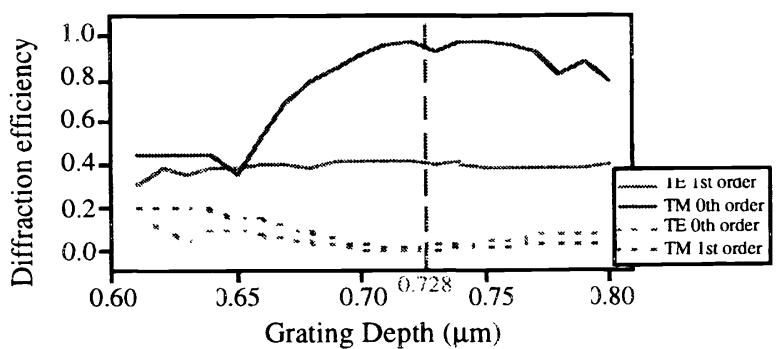

Fig 4. Performance of a binary phase level FBCGH modeled by RCWA.

Applying RCWA to FBCGH elements, we found that the scalar theory (i.e., EMT) can be used to design FBCGH elements accurately, provided that the period of the form birefringent structure is less than $1 / \mathrm{n}$ of the wavelength, where $\mathrm{n}$ is the refractive index of the substrate material. Fig. 4 illustrates the diffraction efficiency of a binary phase level FBCGH as a function of the high frequency grating depth. This simulation result obtained using RCWA shows that the scalar design (depth marked by the vertical line) is ideal to achieve good performances for both polarizations. However, the rigorous coupled wave analysis also shows that a different design can be made to further increase the diffraction efficiency for the TM polarization.

\section{FABRICATION AND CHARACTERIZATION OF MULTIFUNCTIONAL DOES}

These multifunctional DOEs were fabricated using standard microelectronic fabrication technologies. The two substrate BCGH elements and MOD BCGH elements were fabricated similarly: electron beam (E-beam) lithography defines mask patterns, microlithography transfers the pattern into photoresists which are used as etching masks, the surface relief patterns are obtained using ion beam etching with VEECO's microetch system. The argon ions are accelerated at $750 \mathrm{~V}$ and the beam current is $1.3 \mathrm{~mA} / \mathrm{cm}^{2}$. FBCGH elements were fabricated with a slightly modified approach as discussed below.

\subsection{Two substrate BCGH}

We used $\mathrm{LiNbO}_{3}$ as the birefringent substrate to make two substrate BCGH elements. After the surface relieves were etched, the two substrate BCGH elements require an additional assembly process to align and bond the two diffractive structures. This assembly process needs to satisfy the following alignment constraints: (i) parallelism between the two substrates, (ii) minimal separation between the two substrates and (iii) accurate lateral and rotational alignment control between the two diffractive surfaces. We have considered and used two methods. The first method uses existing equipment, such as a standard microlithography mask aligner or a flip-chip bonder; the second method uses an in-situ optical alignment/testing setup constructed on an optical benchtop. We found that the first approach provided a more accurate assembly results. 
Different holograms were designed and fabricated as binary or multi-phase levels. All of the fabricated elements were experimentally evaluated at the designed wavelength in terms of diffraction efficiencies and polarization (or color) contrast ratios (PCRs) at the designed diffraction orders. The diffraction efficiency of the $n$-th diffraction order is defined as the ratio between the output intensity measured at this order and the total diffracted energy,

$$
\eta_{\mathrm{n}}=\frac{\text { Intensity at } \mathrm{n}-\text { th diffraction order }}{\text { Totai transmitted intensity }} \text {. }
$$

This definition excludes the reflection loss from all the surfaces and is more accurate in evaluating the performance of a BCGH element. Diffraction efficiency depends mainly on the number of phase quantization levels, but is also affected by the grating period. The PCR is defined as the ratio of intensities measured in the two orthogonal polarizations at the same diffraction order,

$$
\mathrm{PCR}=\frac{\text { Intensity at desired linear polarization }}{\text { Intensity at the orthogonal polarization }} \text {. }
$$

The best diffraction efficiency achieved by a fabricated binary phase level BCGH element was measured to be $25 \%$ and the highest PCR was better than $40: 1$. For four-phase-level BCGH elements, these performance characteristics increased to about $60 \%$ and 100:1. These efficiencies were significantly less than the theoretical maximum first order efficiency of $40.5 \%$ for a binary phase grating and $80.5 \%$ for a four-phase-level DOE predicted by the scalar diffraction theory. This mismatch is caused by various fabrication errors and inter-pixel crosstalk. With the index matched gap material, a binary phase element demonstrated $40.5 \%$ efficiency and over $400: 1 \mathrm{PCR}^{3}$. To reduce the reflection loss as well as the inter-pixel crosstalk inside a two substrate BCGH element, we coated both sides of the $\mathrm{LiNbO}_{3}$ substrate with antireflection coating. The reflection loss was reduced from $50 \%$ to less than $2 \%$.

\subsection{MOD elements}

$\mathrm{YVO}_{4}$ is the birefringent substrate that we used to fabricate MOD BCGH elements. Due to the multiple order phase delay, the etch depth accuracy required to achieve high efficiency and large PCR is higher than that of a conventional DOE. We made binary phase MOD elements with 39\% measured 1 st order diffraction efficiency and 80:1 PCR. The MOD-based color CGH fabricated in a BK-7 substrate, the first order efficiency was measured to be $39 \%$ with a $30: 1$ contrast ratio.

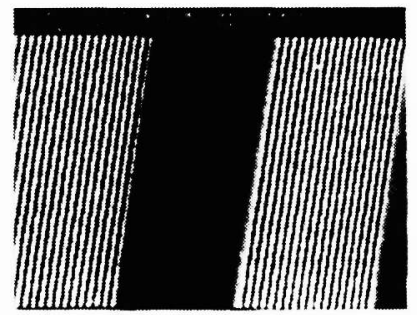

Fig. 5 SEM micrograph of the fabricated binary phase level FBCGH.

\subsection{FBCGH}

We used a (100) cut GaAs substrate to fabricate a diffractive structure using E-beam lithography and dry etching techniques ${ }^{18}$. The pattern was defined using E-beam direct write into PMMA. This pattern was transferred into the GaAs substrate using a three layer process with the aid of ion beam etching, reactive ion etching and chemically assisted ion beam etching. The total area of the fabricated element is $100 \mu \mathrm{m} \times 100 \mu \mathrm{m}$. An SEM micrograph of the fabricated element is shown in Fig. 5. The period of the binary phase diffractive grating (see Fig. 3) $\mathrm{T}$ is $10 \mu \mathrm{m}$. The period of HSFG $\Lambda$ is $0.3 \mu \mathrm{m}$ and the fill factor of the HSFG F is 0.35 . The measure first order diffraction efficiency was over $40 \%$ with $275: 1$ PCR. 


\subsection{Comparison of the three approaches}

The two substrate BCGH is relatively complicated to fabricate. The relatively severe inter-pixel crosstalk with this approach can be overcome by filled the gap between the two substrates with index matching material. Low-index strong-birefringence material such as calcite and $\alpha$-BBO crystal are required for this approach, but they are deliquescent and thus require special handling during the fabrication process. Other natural birefringent materials have large indices of refraction and an index matching fluid cannot be found easily.

The two other approaches used to construct a BCGH element both yield high diffraction efficiencies and large polarization contrast ratios. The FBCGH is more challenging to fabricate, but since the substrate material and the fabrication methods are both compatible with semiconductor technology, FBCGH elements are suitable for packaging optoelectronic devices and systems. The multiple order delay hologram, on the other hand, is relatively easy to fabricate and may find many applications including various optical disc readout head systems. With the advances in microfabrication technologies, both approaches may eventually replace the two substrate approach and may be used in many different applications.

\section{APPLICATIONS}

Multifunctional DOEs are useful in many photonic and optoelectronic applications. Below we describe several specific demonstrated applications examples of such elements.

\subsection{Packaging OE-VLSI chip}

Increasing demand for data transmission and processing has spurred development of silicon VLSI devices with parallel optoelectronic (OE) input and output (I/O). ${ }^{19}$ OE chips with photodetector receivers and laser or optical modulator transmitters can support large-scale high-speed parallel communication over short distances using free space optical interconnects. The optical signals are routed to photodetectors and transmitted from the modulators or laser devices using arrays of DOEs or micro-optics components. In general, these optical input and output signal paths are different, which implies that the optics must provide dual functionality in implementing the communication links. Since conventional DOE or micro-optics components do not have this dual functionality, additional components such as polarization beam splitters are required to separate the input and the output signals. The unique capability of $\mathrm{BCGH}$ elements enables more compact and reliable packaging. The first integration of diffractive beam-forming optics with optoelectronic (L/O) devices flip-chip bonded to CMOS VLSI electronics, and the first integration of a BCGH with any optoelectronic device was reported 5 .

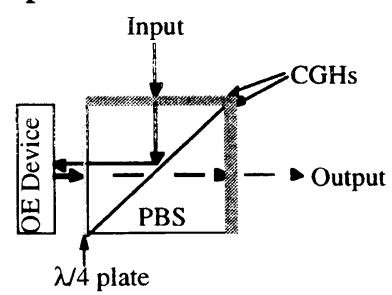

(a)

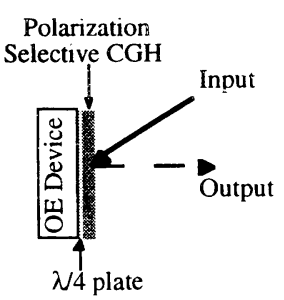

(b)

Fig. 6 Packaging optical interconnects for OE-VLSI (a) conventional approach and (b) BCGH approach.

Fig. 6(a) shows a conventional approach to package optical interconnects with OE VLSI, consisting of a polarization beam splitter, combined with a quarter wave plate and two different conventional DOEs for routing the optical signals. In addition, other bulk optics (not shown in the figure) may be required for input collimation and output imaging. The BCGH package (Fig. 6b) combines the functionality and replaces these three elements as well as removes the need for imaging optics 
because the free space propagation distances are significantly reduced. The principal advantage of this approach is that it replaces a distributed bulk optical system with a compact and rugged OE-VLSI module that has beam forming optics permanently aligned to a chip mounted in a standard electronic ceramic pin grid array (PGA) package. The resultant package is more compact, lighter, and can have better mechanical and thermal stability. Fig. 7 shows a OE-VLSI chip packaged with a binary phase level BCGH constructed using MOD approach. The OE-VLSI chip contained a 20x10 multiple quantum well diode array flip-chip bonded on CMOS VLSI circuit. The packaged module showed uniform modulator illumination and correct spot registration.

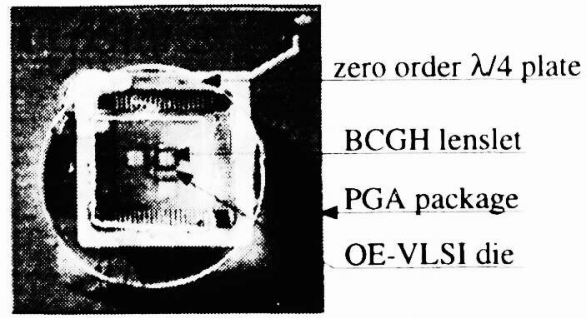

Fig. 7 Photograph of the packaged OE-VLSI module.

\subsection{Packaging transparent optical MIN}

The current trend in high performance computing systems is towards increasing both parallelism and speed, relying on development of novel optical interconnections technology that can support the necessary ultrahigh bandwidths. Based on power and speed considerations, optical links are preferable to electronic wires for "long-distance" communications. ${ }^{20,21}$ In addition to the conventional DOEs that are useful in both all-optical and optoelectronic interconnection networks, BCGH elements offer greater advantages such as simpler packaging configuration and faster routing speed. A BCGH element that has dual impulse responses for the orthogonal linear polarizations can serve naturally as a $1 \times 2$ switch when combined with a polarization modulator. Two BCGH and an electrooptic polarization rotator can be used to construct a binary $2 \times 2$ optical switch. The $2 \times 2$ optical switch is the building block of an optical multistage interconnection network (MIN). A three stage optical MIN was demonstrated using BCGH elements and polarization rotators. ${ }^{22}$

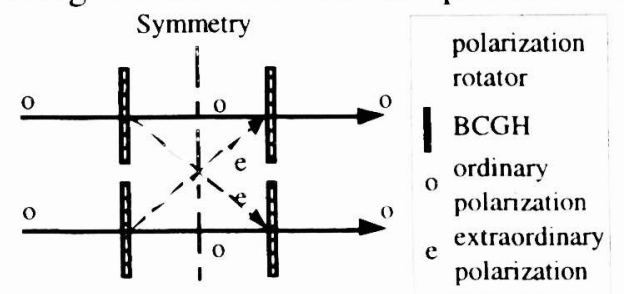

Fig. 8 Dilated bypass-exchange switch.

A more recent attempt at constructing an optical MIN using BCGH elements utilized a 'folded' optical MIN system that permits switching high-speed signals between multiple input and output nodes. Optical routing is performed by dilated bypass-exchange switches (DBES) built of BCGHs combined with electrically addressed ferroelectric liquid crystal device. This scalable system can switch high bandwidth communication lines or permit memory access and multiprocessor interconnections. A schematic of the DBES is illustrated in Fig. 8. Two ordinary polarized optical signals are exchanged when they are transformed into extraordinary polarized light by the polarization rotators. One such switch acts as a $2 \times 2$ interconnection network. By combining several switches into layers, together with simple optics to create a desired interconnection pattern, a larger network can be constructed. It can be seen from Fig. 8 that the DBES is a symmetric switch. By folding the switch along the symmetry line, with the use of a mirror, we can greatly reduce the complexity of the system (see Fig. 9). 


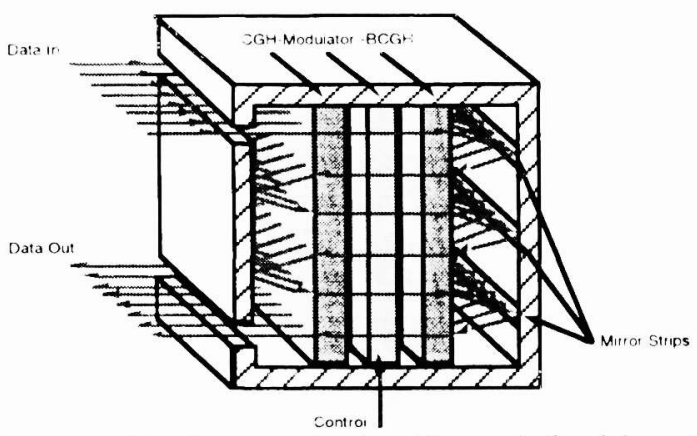

Fig. 9. Folded MIN system layout: Each round trip (from left-side to left-side) completes a single dilated bypass exchange switch. Three passes (as in the above system) allows for $8 \times 8$ interconnection.

Initial testing of a single dilated bypass exchange switch (i.e. $2 \times 2$ system) has shown signal-to-noise ratios ( $\mathrm{SNR}$ ) of $>20: 1$ signal. The signal is generated by an acousto-optic (AO) cell. Since the system is optically transparent, the measured SNR is constant (in the range up to $10 \mathrm{MHz}$ ) and does not depend on the modulating data rate (in contrast to switches with electronic regeneration). Preliminary experimental tests of the system shown in Fig. 9 give an SNR performance of better than 10:1.

\subsection{Wavelength division demultiplexer}

The wavelength selective $\mathrm{CGH}$, described in section 2.2 , was used to demonstrate a wavelength division demultiplexing of $1.3 \mu \mathrm{m}$ and $1.55 \mu \mathrm{m}$ signals using the setup shown in Fig. 10. The output from two fiber-coupled diode lasers was combined into one single-mode fiber by a $2 \times 2$ fiber coupler. The $1.3 \mu \mathrm{m}$ laser was current modulated at $100 \mathrm{~Hz}$, and the $1.55 \mu \mathrm{m}$ laser modulated at $1 \mathrm{kHz}$, for an artificial data signal to distinguish their outputs. The single-mode fibcr output was imaged through the wavelength selective $\mathrm{CGH}$ onto the detector plane where the 0 th, ar \pm 1 st orders could be measured. The oscilloscope trace at the left shows the input light, carrying botn signals. The traces at the center and at the right show the separated outputs at the same scale. The $1.3 \mu \mathrm{m}$ signal is transmitted with negligible cross talk into the 0-th order, whereas the $1.55 \mu \mathrm{m}$ light is deflected with equal efficiency into the $\pm 1 \mathrm{st}$ orders. The ratio of light at wavelength of $1.3 \mu \mathrm{m}$ to that of $1.5 \mu \mathrm{m}$ in the 1-st diffraction order is $30: 1$. The average energy efficiency, including reflections, is $\sim 75 \%$.

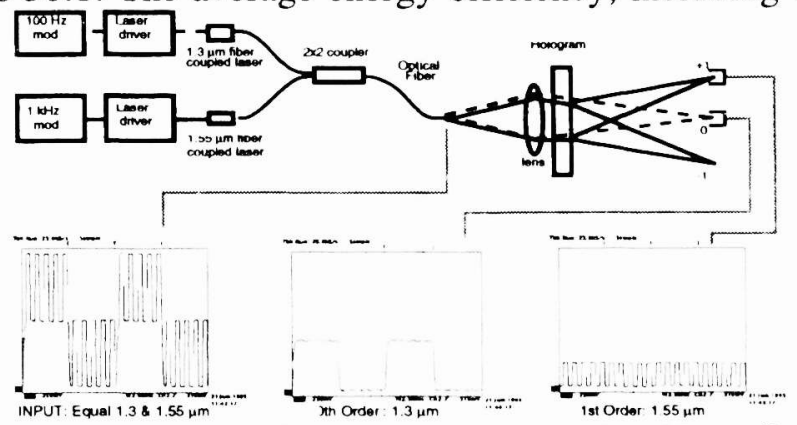

Fig. 10 Wavelength demultiplexing of 1.3 and $1.55 \mu \mathrm{m}$ light signals.
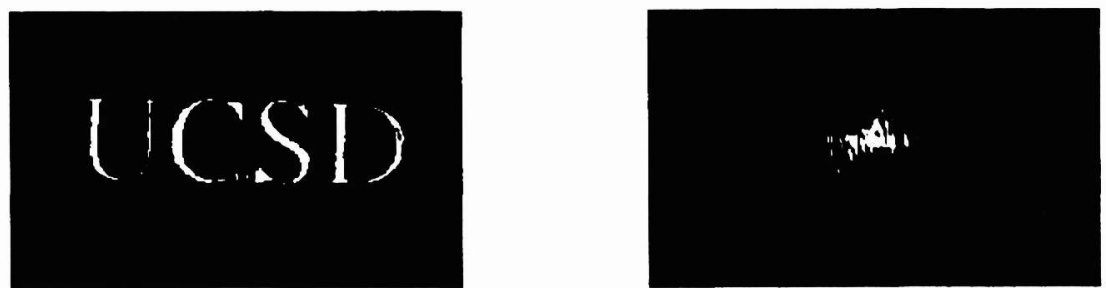

Fig. 11 Image and Fourier Transform of the same object performed by a dual focal length BCGH lens. 


\subsection{Image processing}

By employing a dual focal length BCGH lens with a focal length ratio of two, we can achieve the two important transforms, imaging and Fourier transform at the same plane without any physical motion. ${ }^{23}$ The switching between the two transforms can be obtained by changing the input polarizations (see Fig. 11).

\section{CONCLUSIONS}

We have developed three different approaches to construct diffractive optical elements with multifunctionality in polarization or color. Design methods for these multifunctional DOEs are described for the different approaches. Rigorous vector field modeling tools have been applied to improve and verify the designs obtained with scalar theory. Microfabrication techniques employed to fabricate these multifunctional DOEs are described. Experimental characterization of the various fabricated multifunctional DOEs are reported. Various photonic and optoelectronic applications that benefit from such multifunctional DOEs are described. These applications include packaging optoelectronic devices and systems, transparent photonic multistage interconnection networks, wavelength division demultiplexing, and image processing. These experimental demonstrations prove that multifunctional DOEs in polarization or color are useful and frequently essential for photonic and optoelectronic applications. Our future research is focused on further developing the accurate vector-field design and modeling tools as well as microfabrication techniques to meet the packaging needs of future photonic and optoelectronic devices and systems.

\section{ACKNOWLEDGEMENTS}

Authors thank D. Marom, P. Shames at UCSD and C.C. Cheng at Caltch for helpful discussions. This research was funded in part by NSF, AFOSR, BMDO, DARPA and Air Force Rome Lab.

\section{REFERENCES:}

1 C. W. Haggans, T. K. Fujita, R. K. Kostuk, "Integrated device with diffractive polarization components for a magneto-optical disk head," Proc. SPIE , 1663:46-57, (1992).

2 G. P. Nordin, M. W. Jones, J. H. Kulick, R. G. Lindquist, and et. al., "Three-dimensional display utilizing a diffractive optical element and an active matrix liquid crystal display," Opt. Eng., 35(12):3404-12, (1996).

3 K. S. Urquhart, P. Marchand, Y. Fainman, and S. H. Lee, "Diffractive optics applied to free-space optical interconnects," Appl. Opt., 33(17):3670-82, (1994).

4 K. Rastani. M. Orenstein, E. Kapon and A. Von Lehmen, "Integration of planar Fresnel microlenses with vertical-cavity surface-emitting laser arrays," Opt. Lett. 16, 919, (1991).

5 F. Xu, J.E. Ford, A.V. Krishnamoorthy, and Y. Fainman, "Optoelectronic-VLSI packaging with polarization selective computer generated holograms," Opt. Lett., 22(14):, (1997).

6 Y. Carts, "Rewriteable optical disk drive also reads CD-ROMs," Laser Focus World, 31(1):115, (1995).

7 H. Haidner, P. Kipfer, J. Sheridan, J. Schwider N. Streibl, J. Lindolf, M. Collischon, A. Lang and J. Hutfless, "Polarizing reflection grating beamsplitter for the 10.6- $\mu \mathrm{m}$ wavelength", Opt. Eng., 32(8), 1860 - 1865, (1993).

8 J. Ford, F. Xu, K. Urquhart, and Y. Fainman, "Polarization selective computer generated holograms," Opt. Letts., 18:456-458, (1993).

9 N. Nieuborg, A. Kirk, B. Morlion, H. Thienpont and I. Veretennicoff, "Polarization-selective diffractive optical elements with an index-matching gap material," Appl. Opt., 36(20), (1997).

$10 \mathrm{~S}$. Liu and Y. Chen, "Multilevel binary phase grating polarization device with a birefringent crystal," Opt. Lett., 20:1832-1834, (1995). 
11 I. Richter, P. Sun, F. Xu, and Y. Fainman, "Design considerations of form birefringent microstructures," Appl. Opt., 34:2921-2429, (1995).

12 F. Xu, R. Tyan, J. Ford, and Y. Fainman, "Single substrate polarization selective computer generated holograms," Opt. Letts., 21(7):516-518, (1996).

13 J. Ford, F. Xu, and Y. Fainman, "Wavelength selective planar holograms," Opt. Lett., 21(1):80-82, (1996).

14 F. T. Chen and H. G. Craighead, "Diffractive phase elements based on two-dimensional artificial dielectrics," Opt. Lett., 20(2):121-123, (1995).

$15 \mathrm{~S}$. Aoyama and T. Yamashita, "Grating beam splitting polarizer using multi-layer resist method," Proc. SPIE, 1545:241-250, (1991).

16 D. A. Pommet, M. G. Moharam, E.B. Grann, "Limits of scalar diffraction theory for diffractive phase elements," JOSA A, 11(6):1827-34, (1994).

17 M.G. Moharam, and T.K. Gaylord, "Diffraction analysis of dielectric surface-relief gratings," JOSA, 72:1385-1392, (1982).

18 F. Xu, R. C. Tyan, P. C. Sun, C. C. Cheng, A. Scherer and Y. Fainman, "Fabrication, modeling, and characterization of form-birefringent nanostructures," Opt. Lett., 20:2457, (1995).

19 K. Goossen, J. Walker, L. D'Asaro, S. Hui, B. Tseng, R. Leibenguth, D. Kossives, D. Bacon, D. Dahringer, L. Chirovsky, A. Lentine, D. Miller, "GaAs MQW modulators integrated with silicon CMOS," IEEE Phot. Tech. Lett. 7:360, (1995).

20 M. Feldman, S. Esener, C. Guest, and S. H. Lee, "Comparison between optical and electrical interconnects based on power and speed considerations," Appl. Opt., 27:1742-1751, (1988).

21 D. A. B. Miller, "Optics for low-energy communication inside digital processors: quantum detectors, sources, and modulators as efficient impedance converters," Opt. Lett., 14:146-148, (1989).

22 A.V. Krishnamoorthy, F. Xu, J.E. Ford, and Y. Fainman, "Folarization-controlled multistage switch based on polarization-selective computer generated holograms," Appl. Opt. 36(5):9971010, (1997).

23 F. Xu; J.E. Ford; Y. Fainman, "Polarization-selective computer-generated holograms: design, fabrication, and applications," Appl. Opt., 34(2):256-66 (1995). 